\title{
Visual electrophysiological examination in diagnosis of hepatic encephalopathy ${ }^{*}$
}

\author{
Lei Zhang ${ }^{1}$, Li Li ${ }^{1}$, Xinmin Zhou ${ }^{2}$, Feng Xia ${ }^{1}$, Zuoming Zhang ${ }^{1 \#}$ \\ ${ }^{1}$ School of Aerospace Medicine, Fourth Military Medical University, Xi'an, China \\ ${ }^{2}$ Xijing Hospital, Fourth Military Medical University, Xi'an, China \\ Email: ${ }^{*}$ zhangzm@,fmmu.edu.cn
}

Received 31 January 2013; revised 8 March 2013; accepted 5 April 2013

Copyright (C) 2013 Lei Zhang et al. This is an open access article distributed under the Creative Commons Attribution License, which permits unrestricted use, distribution, and reproduction in any medium, provided the original work is properly cited.

\begin{abstract}
Clinical observation of the neuropsychiatric condition, psychometric tests, and EEG recordings has been used to diagnose hepatic encephalopathy. However, no standardization for the diagnosis of minimal hepatic encephalopathy (MHE) has been achieved. According to the study in normal subjects, visual electrophysiology testing is a method of simple, objective record for the medical examination, which includes visual evoked potentials (VEP), electroretinograms (ERG), etc. 23 hepatic encephalopathy patients prospectively underwent visual electrophysiology testing and laboratory serum analysis, and it was found that 22 visual electrophysiology abnormalities which showed that the amplitudes were decreased and the implicit times were prolonged compared with the normal. The relationship between biochemical parameters and visual electrophysiology parameters showed that visual recording was related to the damage of the liver. On the basis of this preliminary study, we could conclude that this method can be as a way to predict for hepatic encephalopathy and it appears to be a method in the recognition of the MHE.
\end{abstract}

Keywords: Hepatic Encephalopathy; VEP; ERG; Visual Electrophysiological Examination; Diagnosis

\section{INTRODUCTION}

Hepatic cirrhosis due to hepatitis and intoxication may result in a neuropsychiatric syndrome called hepatic encephalopathy (HE), and the subclinical HE called minimal hepatic encephalopathy (MHE) is highly prevalent (22\% - 74\%) among patient with liver dysfunction [1]. MHE has no clinical evidence of hepatic encephalopathy,

\footnotetext{
"Supported by Xijing Hospital, Fourth Military Medical University.

${ }^{\#}$ Corresponding author.
}

and neuropsychological tests and another especial measurement like evoked potentials and image studies can only make diagnosis. Diagnosis of MHE may have prognostic and therapeutic implications in cirrhotic patients [2].

In patients with liver insufficiency the blood ammonia concentration is elevated, which induces structural and functional retinal alterations referred to as "hepatic retinopathy" [3]. More than 100 years ago [4], Alzheimer described changes in the brains of patients who had suffered hepatic failure. Astrocytes are primarily affected because they are the only type of cells in the brain capable of detoxifying ammonia [5]. Embryologically, the retina is part of the brain. The dominating retinal microglia, the Müller cells, share many properties with astroglia of the brain. Thus, it concluded that increased levels of serum ammonia resulting from hepatic insufficiency cause changes in Müller cells that are similar to brain astrocytes [4]. As disturbances of Müller cell functions may, directly or indirectly, contribute to visual disabilities observed in patients with liver diseases, the term "hepatic retinopathy" has been introduced in analogy to "hepatic encephalopathy" [6].

Visual electrophysiology is a method of simple, objective record for the medical examination, which includes visual evoked potentials (VEPs), electroretinograms (ERG), etc. VEPs were first used in detecting abnormalities of the visual system [7] and of many neurological diseases with visual symptoms. They were also utilized in the evaluation of uraemic coma. In fact, VEP recordings have been used in animals with fulminant hepatic failure, and also in-patients with hepatic encephalopathy and acute severe hepatitis [8]. However, because VEPs reflect the resulting response of the cortex after the stimulation of visual areas and can be affected by retinal disease not only cortical, some studies seem that VEPs are not as sensitive as somatosensory evoked potentials (SEP) [9] and have not been widely used. ERG has not 
been used in the diagnosis of HE.

As "hepatic retinopathy" has been introduced in analogy to "hepatic encephalopathy" and Müller cell functions contribute to changes of the ERG, we suggest the diagnosis of HE can use combination of VEP and ERG to improve the sensitivity, especially for MHE. Susann et al. [5] had used ERG as the method of comparing functional changes of the retina before and after liver transplantation to evaluate the postoperative improvement. The aim of this investigation was to evaluate the diagnostic value of the visual electrophysiology testing as an objective measurement of $\mathrm{HE}$ in liver cirrhosis patients and possibly as a test for recognition of MHE.

\section{MATERIALS AND METHODS}

\subsection{Subjects}

Between October 2007 and June 2008, 29 liver cirrhosis patients prospectively underwent visual electrophysiology testing and laboratory serum analysis (23 male, 6 female, mean age $51 \pm 9.83$ years). 23 of these patients showed symptoms of encephalopathy (Child-Pugh A, B, C: $5,10,8)$; 4 of them were tested visual electrophysiology again after receiving therapies which was artificial liver support system (ALSS). The cause of liver cirrhosis was very heterogeneous in our study (2/29 alcoholic, 17/29 hepatitis B). Patients were tested by visual electrophysiology within one hour and venous blood samples were collected at the same time as the tests. Informed consent was given all subjects before the testing.

The diagnosis of liver cirrhosis was based on biochemical, oesophagogastroscopic findings and on liver biopsy. Diagnosis of HE was performed by experienced clinical surgeons. HE was diagnosed according to guidelines in the final report of the Working Party at the 11th World Congresses of Gastroenterology in Vienna in 1998. The neuropsychologic test battery included number connection tests $\mathrm{A}$ and $\mathrm{B}$ and the digit symbol test.

\subsection{Parameters}

Laboratory parameters included ALT (U/1), AST (U/1), serum total bilirubin $(\mu \mathrm{mol} / \mathrm{l})$, albumin $(\mathrm{g} / \mathrm{l})$, prothrombin time $(\mathrm{S})$ and ammonia $(\mathrm{g} / \mathrm{l})$.

Visual electrophysiology testing included VEP and ERG. The VEP parameters which we evaluated were amplitudes and latencies of P100-wave after pattern stimulation (P-VEP) and P1-wave after flash stimulation (FVEP). The ERG parameters which we evaluated were rod, standard combined response (SC) and cone amplitudes and implicit times of a- and b-waves after single flash stimulation, the amplitudes of oscillatory potentials (Ops) and $30 \mathrm{~Hz}$ flicker stimulation. The measurements were performed according to the standards of the International Society for Clinical Electrophysiology of Vision.
The normal values were established in our laboratory using the below mentioned equipment in 43 healthy volunteers of mean age $50 \pm 7.37$ years group.

Patients were light-adapted at first. Two $\mathrm{AgCl}$ electrodes were applied to the scalp with collodium. The active electrode was placed at $\mathrm{Oz}$ and the reference electrode was placed at Fz. The right earlobe was grounded. Interelectrode impedance was reduced to $<5 \mathrm{~K} \Omega$ with light abrasion of the scalp, using salt gel as a conducting medium. For the pattern-VEP the retina was stimulated reversally with a black and white checkerboard in a white background illumination. Then for the flash-VEP patients were stimulated in the dark with an automated Ganzfeld stimulator which was the same as ERG. Patients' pupils were fully dilated with $0.5 \%$ tropicamide. After 20 min of dark adaptation, the scotopic ERG responses were recorded. Prior to the recording of the photopic ERG, the patients were light-adapted $\left(20 \mathrm{~cd} / \mathrm{m}^{2}\right)$ for $10 \mathrm{~min}$. ERG were obtained by placing an active electrode on the cornea, embedded in a corneal contact lens. The reference skin electrode was placed on the external angle, the ground electrode on the right earlobe.

All the testings were used the GT-2000NV system (made in China) according to the International Society for Clinical Electrophysiology of Vision.

\subsection{Data Analysis}

All data were presented as means \pm SD. Student's $t$-test and canonical correlation analysis were used to analyze the data. The criterion for significance was $P<0.05$ in all experiments.

\section{RESULTS}

\subsection{Visual Electrophysiology Parameters}

23 patients showed symptoms of encephalopathy in clinic and visual electrophysiology abnormalities were found in 22 cases (see Table 1). Figure 1 One case studied first without symptoms of encephalopathy in clinic was abnormal in visual electrophysiology parameters, and subsequently showed hepatic encephalopathy three days later. Two patients with symptoms at prodromal stage of HE recorded in normal of visual electrophysiol-

Table 1. The early diagnosis of hepatic encephalopathy by visual electrophysiology.

\begin{tabular}{cccc}
\hline \multirow{2}{*}{ visual electrophysiology } & \multicolumn{2}{c}{ The symptoms at prodromal stage } \\
\cline { 2 - 4 } & + & - & total \\
\hline+ & 21 & 1 & 22 \\
- & 2 & 5 & 7 \\
total & 23 & 6 & 29 \\
\hline
\end{tabular}




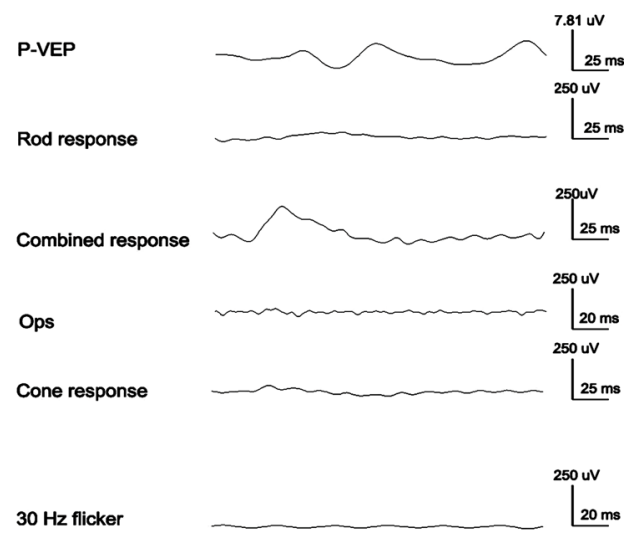

(a)
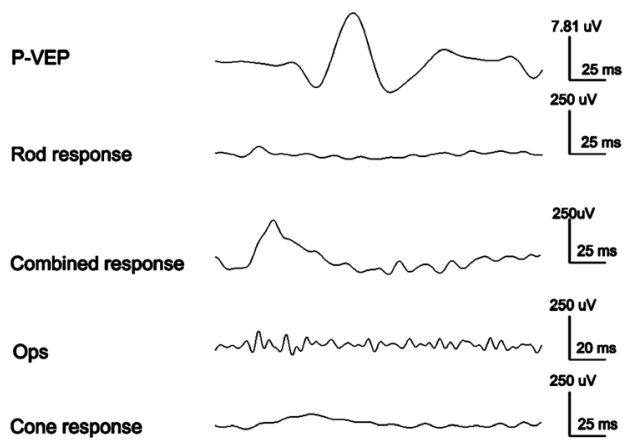

$30 \mathrm{~Hz}$ flicker

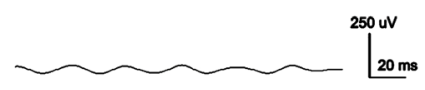

(b)

Figure 1. Patterns of visual electrophysiology recorded in one patient without HE (a) and in one patient with encephalopathy (b); (a) Although the patient didn't show any symptoms of encephalopathy, the results of VEP and ERG displayed that the time of visual transmission was prolonged and the function of the retina was declined. And the patient showed hepatic encephalopathy three days after the examination; (b) The patient with encephalopathy had normal VEP and ERG, and recovered well after therapy.

ogy parameters. Figure 2 the patient studied first at prodromal stage of HE, was subsequently examined when he was treated with artificial liver support system (ALSS).

The amplitudes of the SC, cone b-waves, the SC awave, the P100 and the P1 and the implicit times of the cone b-wave, the P100 and the P1 were out of the normal range as defined in our laboratory (see Figures 3 and 4). It was showed that the amplitudes were decreased and the implicit times were prolonged compared with the normal.

\subsection{Biochemical Parameters}

Canonical correlation analysis was used to study the re-

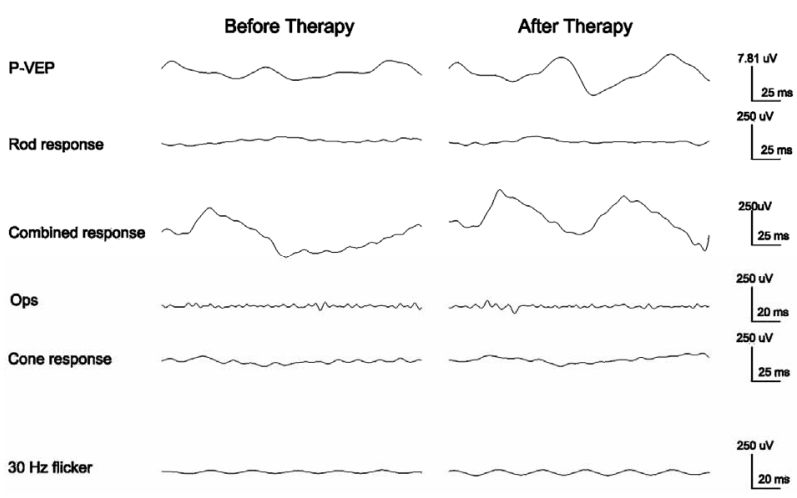

Figure 2. The patient was treated with ALSS. After therapy the results of VEP and ERG showed that the amplitudes were increased and the latencies were shortened. The patient recovered well and then discharged from hospital a week later.

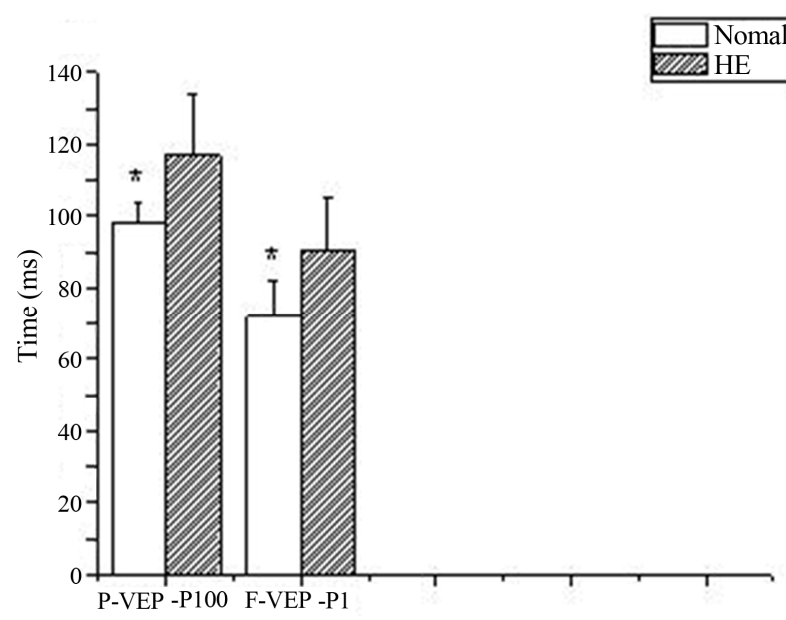

(a)

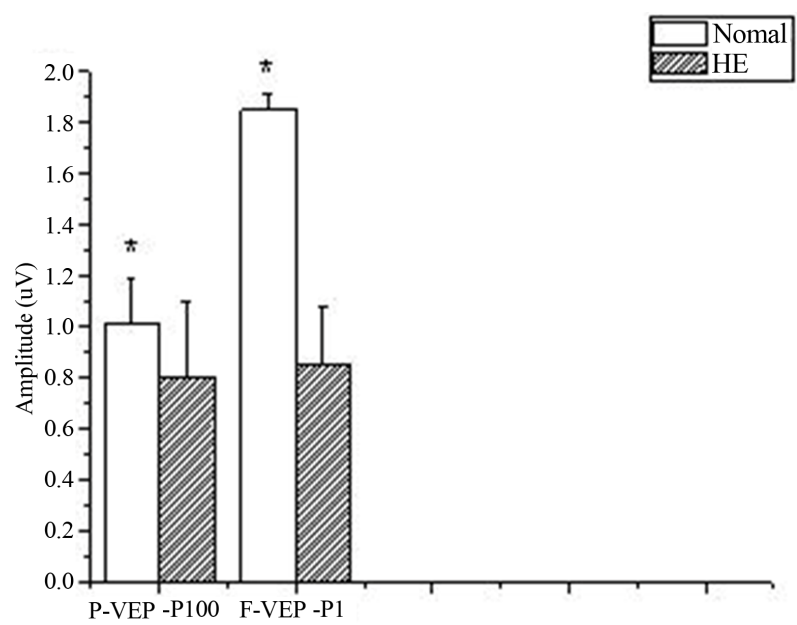

(b)

Figure 3. VEP parameters assessed between the normal and HE: (a) The latencies of P-VEP P100 and F-VEP P1 were longer than the normal; (b) The amplitudes of P-VEP P100 and F-VEP P1 were decreased compared with the normal. Student's $t$ test: ${ }^{*} P<0.05$ vs normal. 


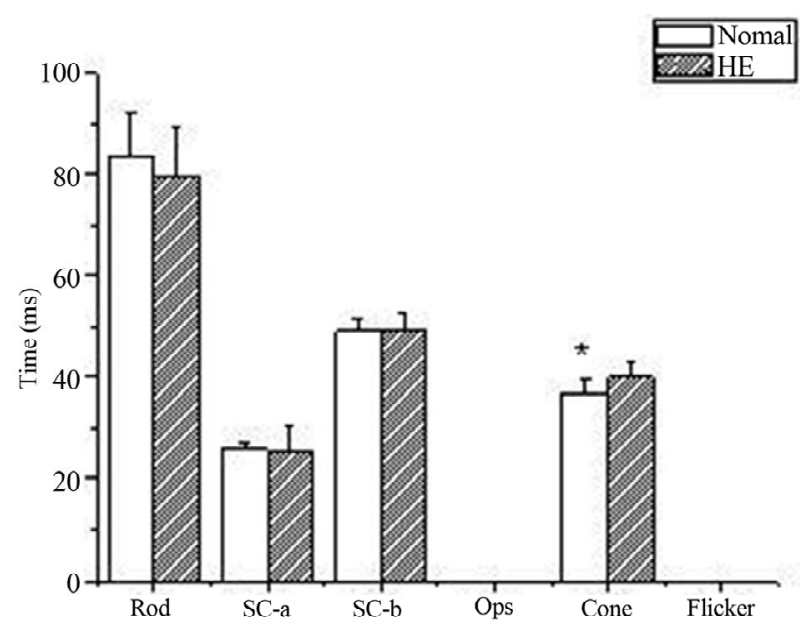

(a)

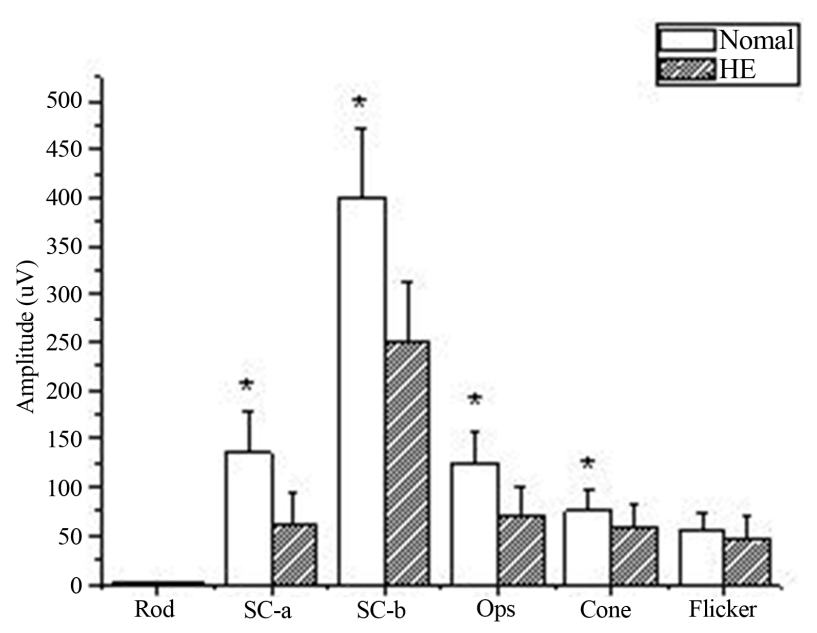

(b)

Figure 4. ERG parameters assessed between the normal and HE: (a) The implicit time of the cone b-wave was longer than the normal; (b) The amplitudes of the SC, cone b-waves, the $\mathrm{SC}$ a-wave and Ops were decreased compared with the normal. Student's $t$ test: ${ }^{*} P<0.05$ vs normal.

lationship between biochemical parameters and visual electrophysiology parameters. Biochemical parameters included ALT (X1), AST (X2), serum total bilirubin (X3), albumin (X4), prothrombin time (X5) and ammonia (X6). Visual electrophysiology parameters included the amplitudes of SC b-wave (Y1), cone b-wave (Y2), Ops (Y3) and P-VEP P100 (Y4) and the latencies of cone b-wave (Y5) and P-VEP P100 (Y6). According to F test of canonical coefficient correlations $(F=3.95, p=$ $0.0017)$, a pair of standardized canonical correlation variances was gotten as below.

$$
\begin{aligned}
\mathrm{U} 1= & -0.5734 \mathrm{X} 1+1.0004 \mathrm{X} 2-0.0857 \mathrm{X} 3 \\
& -0.1773 \mathrm{X} 4-0.3288 \mathrm{X} 5-0.5361 \mathrm{X} 6 \\
\mathrm{~V} 1= & 0.4338 \mathrm{Y} 1+0.9674 \mathrm{Y} 2+0.6233 \mathrm{Y} 3 \\
& +0.2806 \mathrm{Y} 4+0.0362 \mathrm{Y} 5-0.4852 \mathrm{Y} 6
\end{aligned}
$$

From the weight numbers of canonical correlation variances, it was showed that U1 was mainly affected by ALT, AST and ammonia and V1 was mostly decided by amplitudes of cone b-wave, Ops and SC b-wave and the latency of P-VEP P100. According to the equation, the values of ALT, AST and ammonia were related to the amplitudes of cone b-wave, Ops and SC b-wave and the latency of P-VEP P100.

\section{DISCUSSION}

So far, no standardization for the diagnosis of MHE has been achieved. According to the study in normal subjects, visual electrophysiology testing is a reliable method and waveforms are constant. This method proved objective and sensitive enough in diagnosis of HE. It was found 22 visual electrophysiology abnormalities in $23 \mathrm{HE}$ patients while visual electrophysiology testing didn't know idea of the clinical diagnosis. One visual electrophysiology normal of HE case recovered very well suggested by biochemical parameters and the clinical observation and then discharged from hospital. On the basis of this case and the comparison of before and after the therapy, maybe we could conclude that this method can be as a way to predict for HE. Another important point in favour of the visual electrophysiology testing is the fact that it appears to be a method in the recognition of the subclinical stage of hepatic encephalopathy. With this method we found one patient who appeared normal to neuropsychiatric examination. Biochemical parameters and the observation of a clear encephalopathic state developed three days later suggested that he was in a subclinical stage of hepatic encephalopathy when tested.

According to the standards of the International Society for Clinical Electrophysiology of Vision, each laboratory needs to establish normal values for its own equipment [10]. We compared visual electrophysiology recording of patients with the normal range as defined in our laboratory. It was showed that the amplitudes were decreased and the implicit times were prolonged compared with the normal. In fact, VEP was used to assess HE in our department in 1990s [11]. However, ERG has not been used to test HE. From our study of the ERG parameters of $\mathrm{HE}$, the amplitudes were decreased significantly which showed that their retinal function was weakened which was called "hepatic retinopathy". After timely normalization of the metabolic state of the patients, the ERG parameters of patients show a significant recovery [5]. This means that we may use the ERG to observe the recovery of hepatic retinopathy as the therapeutic/preventive approaches of HE. And the ERG and VEP should be used together to assess for HE from visual transmission and retinal function.

The relationship between biochemical parameters and visual electrophysiology parameters showed that visual 
recording was related to the damage of the liver. The more liver cells were injured, the amplitudes of the ERG parameters were decreased and the latency of the VEP parameters was prolonged. Visual electrophysiological testing seemed to be a suitable method for evaluation of the different degrees of encephalopathy.

In summary, on the basis of this preliminary study visual electrophysiological examination was an objective method for diagnosis and prognosis of HE. The advantages of it could therefore be of service in the follow up of patients to prevent the occurrence of $\mathrm{HE}$ and monitoring inpatients during therapy. It was also hoped that visual electrophysiological examination could be used for model animal research to find the pathogenesis of hepatic encephalopathy.

\section{REFERENCES}

[1] Zhan, T. and Stremmel, W. (2012) The diagnosis and treatment of minimal hepatic encephalopathy. Deutsches Ärzteblatt International, 109, 180-187.

[2] Zamora, N.L. and Torre, D.A. (2011) Minimal hepatic encephalopathy. Annals of Hepatology, 10, S50-S54.

[3] Kuhrt, H., et al. (2004) Rabbit retinal organ culture as an in-vitro model of hepatic retinopathy. Graefe's Archive for Clinical and Experimental Ophthalmology, 242, 512522. http://dx.doi.org/10.1007/s00417-004-0882-2

[4] Reichenbach, A., et al. (1995) Hepatic retinopathy: Morphological features of retinal glial (Muller) cells accompanying hepatic failure. Acta Neuropathologica, 90, 273281. http://dx.doi.org/10.1007/BF00296511
[5] Uhlmann, S., Uhlmann, D., Hauss, J., Reichenbach, A., Wiedemann, P. and Faude, F. (2003) Recovery from hepatic retinopathy after liver transplantation. Archive for Clinical and Experimental Ophthalmology, 241, 451-457. http://dx.doi.org/10.1007/s00417-003-0639-3

[6] Scholl, H.P. and Zrenner, E. (2000) Electrophysiology in the investigation of acquired retinal disorders. Survey of Ophthalmology, 45, 29-47. http://dx.doi.org/10.1016/S0039-6257(00)00125-9

[7] Halliday, A.M., McDonald, W.I. and Mushin, J. (1973) Delayed pattern evoked responses in optic neuritis in relation to visual acuity. Transactions of the Ophthalmological Societies of UK, 93, 315-324.

[8] Zamir, D., et al. (2002) Early detection of hepatic encephalopathy by recording visual evoked potential (VEP). Roczniki Akademii Medycznej w Bialymstoku, 47, 186193.

[9] Zhu, Q., Yang, Y.L. and Wang, M.J. (1993) Diagnosis of subclinical hepatic encephalopathy: A parallel and comparative study between the evoked potentials and the performance psychometric tests. Zhonghua Nei Ke Za Zhi, 32, 676-678.

[10] Michael, F.M., Graham, E.H., Mathias, W.S. and Yamamoto, S. (2004) Standard for clinical electroretinography (2004 update). Documenta Ophthalmologica, 109, 107114.

[11] Miao, J.Y., Pan, B.R., Guo, S.Y., et al. (1990) The relationship between VEP and HE in liver cirrhosis. Medical Journal of Chinese PLA, 15, 113-115. 